\section{Nanofilms on a hollow core fiber}

\author{
Ignacio R. Matias, Javier Bravo, Francisco J. Arregui, \\ and Jesús M. Corres \\ Universidad Pública de Navarra \\ Departamento de Ingenieria Electrica y Electronica \\ Campus de Arrosadia \\ 31006 Pamplona, Spain \\ E-mail: natxo@unavarra.es
}

\begin{abstract}
We experimentally study the behavior of one multimode fiber-hollow core fiber-multimode fiber structure when nanofilms are deposited on it with the aim of developing practical evanescent field-based devices, such as sensors, filters, etc. The electrostatic self-assembly (ESA) method is used as the deposition technique and the chosen polymers are PDDA and Poly R-478 because of their wellknown optical properties and their potential application as humidity sensors. Three different types of hollow core, fibers are used for the fabrication of the devices and at two different wavelengths. An oscillatory-decreasing transmitted optical power is obtained as the thickness of the nanofilms is increased. (c) 2006 Society of Photo-Optical Instrumentation Engineers. [DOI: 10.1117/1.2196043]
\end{abstract}

Subject terms: hollow core fibers; optical fiber devices; nanofilms.

Paper 051000LR received Dec. 30, 2005; revised manuscript received Feb. 6, 2006; accepted for publication Feb. 24, 2006; published online May 2, 2006.

\section{Introduction}

There is an increasing interest in the development of novel types of special fibers for optical devices. ${ }^{1,2}$ Some examples are holey fibers (photonic band gap fibers, indexguiding photonic crystal fibers, Bragg fibers), omniguide fibers, and hollow core fibers ( $\mathrm{HCF}$ ). New applications, such as fiber-optic strain sensors, ${ }^{3}$ broad band bandpass filters, ${ }^{4}$ electrically controllable long-period liquid crystal fiber gratings, ${ }^{5}$ or mode converters ${ }^{6}$ using these fibers have been already proposed using these nonconventional guiding structures. Furthermore, there are many fabrication possibilities for each one, and probably the simplest ones are HCF. Different HCFs have been fabricated. ${ }^{1}$ For simplicity, only the design with a central air hole and silica cladding is selected in this work.

We have studied the influence of a nanofilm deposited onto a simple HCF-based structure that consists of a shortlength HCF segment spliced between two standard multimode fibers (MMF), which will be designated MHM through the text for the sake of simplicity. The nanofilm is deposited using the ESA technique, which allows control over the thickness of the coating on the order of few nanometers. $^{7}$

Previous works have reported applications of similar structures, ${ }^{3-6}$ but in this work we focus our attention on the change experienced in the transmitted power characteristics of the light guided through the MHM as the thickness of the deposited film is increased. As will be reported, this change depends on several parameters. Some of them will

0091-3286/2006/\$22.00 @ 2006 SPIE be experimentally analyzed toward the development of practical evanescent field devices. This structure has been chosen because of its simplicity, sensitivity, robustness, and low cost. The use of a plastic cladding fibers (PCF) instead of HCF yields to a lower sensitivity structure, ${ }^{8}$ and the main problem with tapered fibers is their bending vulnerability, ${ }^{9}$ just to mention a couple of evanescent-fieldbased alternative arrangements already implemented. Other advantages include flexibility in the design (diameters and length of the HCF) and potential applications. ${ }^{1}$

The remainder of this paper is organized as follows. In Sec. 2 the design and fabrication arts are explained. Later on, the experimental setup developed to characterize this nanostructure together with the technique used to control the thickness of the nanodeposition is detailed. In Sec. 4 there is an analysis of results obtained. Finally, some concluding remarks are given.

\section{Fabrication of the MHM}

The structure of the device proposed in this work is shown in Fig. 1(b). It consists of one short segment (around $15 \mathrm{~mm}$ ) of HCF spliced between two MMF. Previously, the jacket of the HCF had to be removed. If the HCF and the MMF are spliced together using the appropriate electric arc conditions, the HCF collapses and forms a tapered solid fiber in the interface between both fibers [see Fig. 1(a)]. In these devices, the light that is guided in the core of the lead-in MMF can be coupled to the cladding of the HCF due to the tapered region, instead of being confined in the air core. When the light reaches the lead-out MMF, it will be coupled into the silica core again. Because the light is guided by the silica cladding in the HCF region, these devices can be used as evanescent field fiber devices. In Fig. 1(c)-1(e) we show some pictures of the light projected by the MHM structure when it is cleaved by different sections. As can be seen, first the light reaching the HCF section is

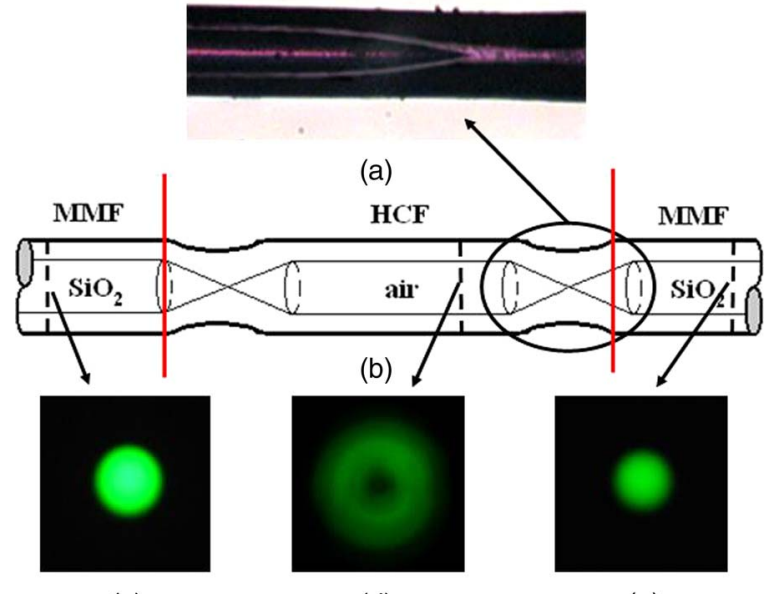

(c)

(d)

(e)

Fig. 1 (a) Photograph of the collapsed end of the HCF spliced to the lead-out MMF section. (b) Scheme of the MHM. (c), (d), and (e) Pictures of the light projected by different parts of the MHM on a screen, using a LED at $480 \mathrm{~nm}$. (c) Light projected by the lead-in MMF. (d) Light in the HCF section and (e) light leaving the lead-out MMF. The positions where the fiber was cleaved are indicated with a vertical solid line. 
guided into the core; then, in the $\mathrm{HCF}$ section, the light becomes guided by the cladding and, finally, the light is guided again by the lead-out MMF core.

\section{Experimental Setup}

\subsection{Controlling the MHM Coating Thickness}

Figure 2 shows the experimental setup used. The light of two superluminiscent LEDs at 1310 and $850 \mathrm{~nm}$ is multiplexed with one tapered optical fiber 50/50 coupler into one fiber and guided through the lead-in MMF. Then, it passes through the HCF cladding and goes to the lead-out MMF that is connected to an $850 / 1310$ demultiplexer. This way, the MHM response to each wavelength can be obtained separately with the optical detector and recorded in a computer.

Along with the MHM device and at the same time, a control fiber whose end has been cleaved perpendicular to its axis is placed as shown in Fig. 2. The control fiber used was a standard MMF coupled to a nanoFPTM module (from Nanosonic, Inc.), which integrates an 850-nm LED source, a 50/50 coupler, and an optical detector. This way, a thin film is being created onto the cleaved end-face of the optical fiber as the material is deposited using the ESA technique creating a nano-Fabry-Perot interferometer, at the same time that the coating is growing onto the MHM-based structure. Analyzing the curve obtained from this interferometer (shown at the top of Fig. 2), it is possible to infer the thickness and the refractive index of the material deposited. ${ }^{10}$ In this case, each bilayer was around $15 \mathrm{~nm}$ thick and the refractive index was approximately 1.53 . The main advantage of this thickness-control technique is that the monitoring can be carried out during the MHM fabrication process.

\subsection{ESA Technique}

ESA has been demonstrated to be a good method to deposit thin layer films of many different materials on the nanometers scale. ${ }^{8,10}$ The technique takes advantage of the electrostatic attraction between polyanionic and polycationic molecules. The solutions selected for the experiments described in this work were Poly R-478 (anthrapyridone chromophore) as the anionic electrolyte and poly (diallyldimethyl ammonium chloride) (PDDA), as the cationic

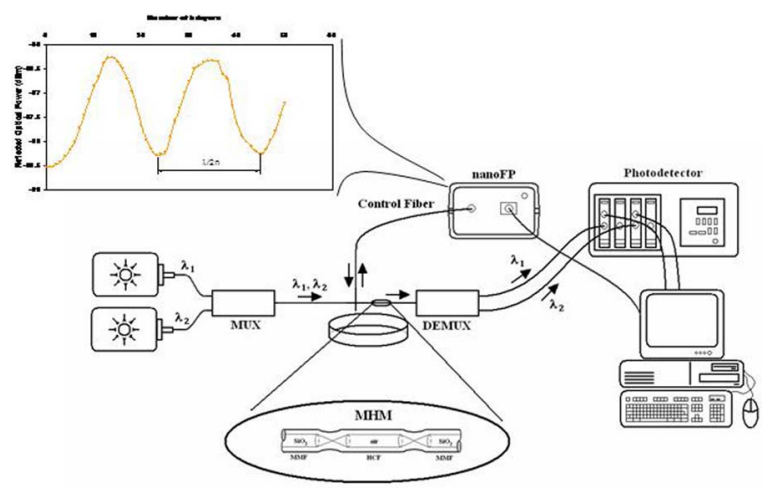

Fig. 2 Experimental setup. The interferometric signal obtained from the control fiber (optical power versus number of bilayers) and used to calculate the thickness deposited on the MHM structure is indicated at the top.

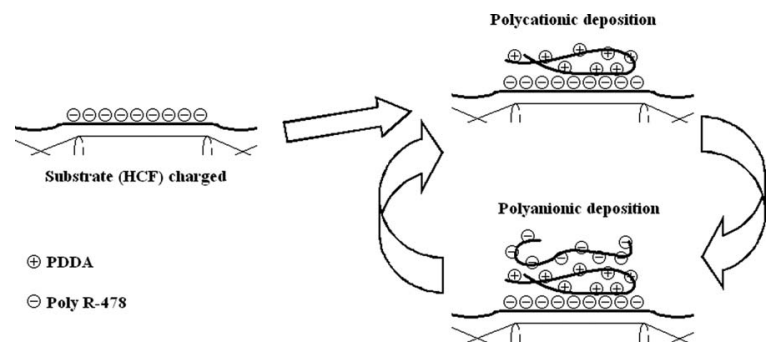

Fig. 3 ESA method protocol.

solution. In previous works it has been demonstrated that this combination behaves as an homogeneous optical film with interesting humidity sensing properties. ${ }^{10}$ Before proceeding to the deposition, the substrate surface (the HCF external surface) has to be electrostatically charged. If this charge is negative the cationic material will be deposited first. In this case, the substrate was cleaned and charged negatively, therefore the substrate was dipped first into the cationic solution for enough time to let the electrostatic forces act and get an optical uniform layer added to the substrate. Then, it is dipped into deionized water in order to wash it. The next step consists of repeating the same procedure, but this time using the anionic solution. This process is repeated (Fig. 3), increasing, layer by layer, the thickness of the material deposited onto both the MHM structure and the control fiber.

\section{Experimental Results}

Some experimental results are presented in Figs. 4 and 5. In Fig. 4, the HCF segment has a length of $15 \mathrm{~mm}$ and the inner and outer diameters are 102 and $160 \mu \mathrm{m}$, respectively. In Fig. 5 the HCF segment used in the MHM has a length of $20 \mathrm{~mm}$, and diameters of $50 / 150 \mu \mathrm{m}$.

The power transmitted by MHM structures follows an oscillatory path as the number of nanobilayers deposited gets increased. As it can be seen, the period of this oscillation depends, among other parameters, on the wavelength of the light source as expected due to the modal interferometer behavior it exhibits. ${ }^{2}$ Furthermore, it is observed a gradual decrease of transmission due to the nonzero imaginary part of the refractive index of the nanofilm deposited. The different slope in both figures is also noticeable; in fact, there are almost $10 \mathrm{~dB}$ of difference between the first

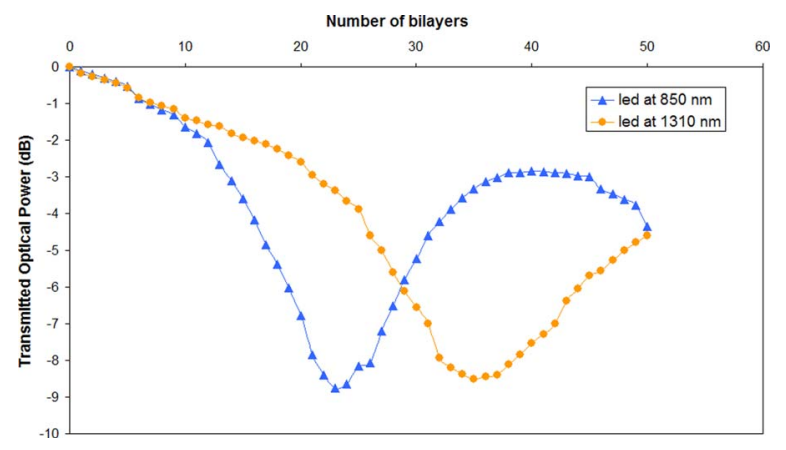

Fig. 4 Power transmitted by a MHM structure. HCF of 102/160 $\mu \mathrm{m}$ inner and outer diameters, respectively. HCF segment length: $15 \mathrm{~mm}$. 


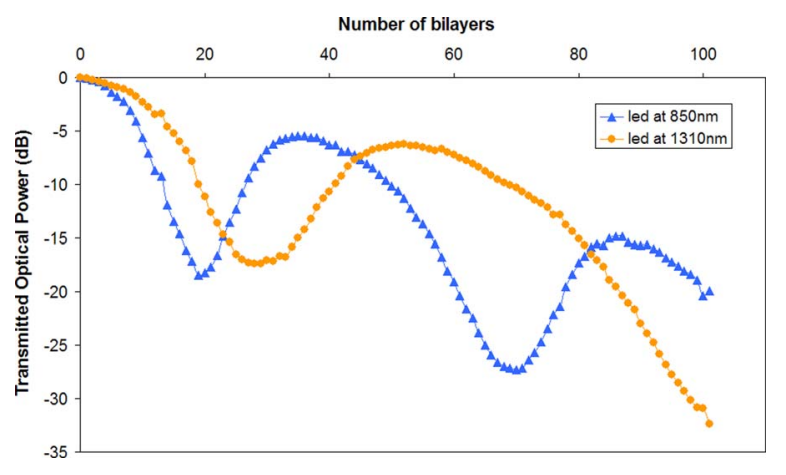

Fig. 5 Power transmitted by a MHM structure. HCF of $50 / 150 \mu \mathrm{m}$ inner/outer diameters, respectively. HCF segment length: $20 \mathrm{~mm}$.

output optical power minimum achieved in the curves of Fig. 5 with respect to the one achieved in Fig. 4. This is due to the different geometrical characteristics of the HCF segment used. In order to clarify the influence of the inner diameter of the HCF segment used, we obtained the experiment in Fig. 6. The HCFs used have inner diameters of 10 and $50 \mu \mathrm{m}$. The outer diameters and the total length in both cases have been fixed to $15 \mathrm{~mm}$ and $150 \mu \mathrm{m}$, respectively. Realize that the amplitude of the oscillatory behavior of the transmitted power tends to reduce when the inner diameter decreases because the evanescent field ratio with respect to the total transmitted optical power is higher.

Other parameters exist that also affect the optical power transmission of this device such as the length of the HCF section, the splice machine parameters used to taper the multimode fibers (that is, the slope of the taper), the index of refraction of the overlay, etc. Because of this, a thoroughly theoretical study covering all these effects will be published elsewhere.

Finally, in order to demonstrate the ability of the proposed structure to sense the surrounding environment, and taking into account that the overlay is humidity sensitive, the MHM structure was introduced into a climatic chamber where a control signal is applied (dotted line in Fig. 7), keeping the temperature at a constant ambient value. The experimental curves obtained are shown in Fig. 7. It can be seen that the structure behaves as a humidity sensor with an apparently noisy signal, probably due to the climate chamber. Also, the response of the sensor seems to be repetitive.

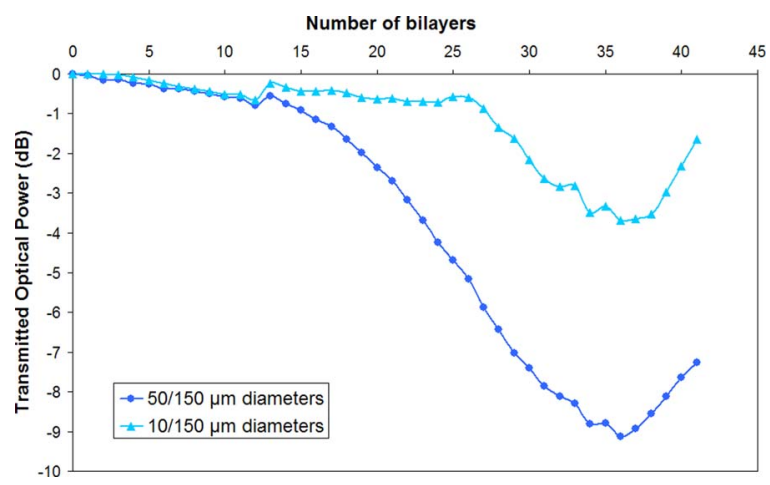

Fig. 6 Power transmitted by two MHM structures $15 \mathrm{~mm}$ long at $1310 \mathrm{~nm}$. HCF of 10/150 and 50/150 $\mu \mathrm{m}$ inner and outer diameters, respectively.

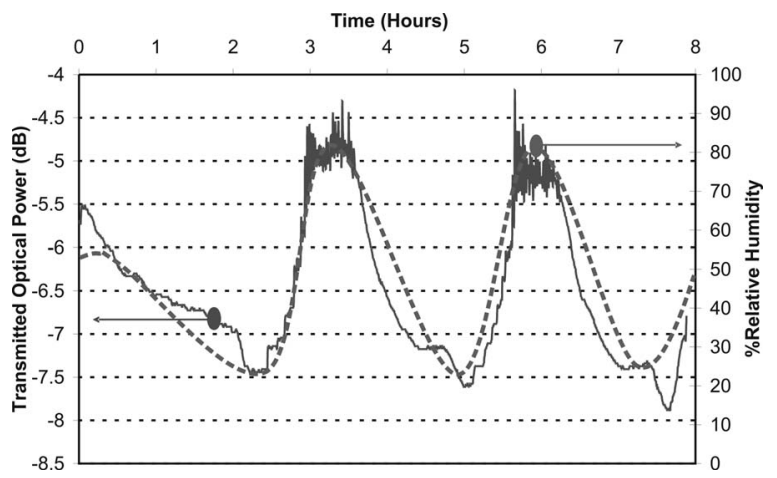

Fig. 7 Humidity response of a MHM structure at a wavelength of $1310 \mathrm{~nm}$. Dotted line is the control signal applied to the climatic chamber. Solid line is the response of the MHM. Overlay: $[\text { PDDA }]^{-} /[\text {PolyR478 }]^{+}$.

\section{Conclusions}

The behavior of MHM-based structures at two different wavelengths using different HCF segments when a nanofilm is being deposited on them has been experimentally analyzed. The power transmitted follows an oscillatory path whose period and depth depends on the wavelength used and the geometrical characteristics of the HCF utilized, respectively. These results could be used to optimize prior applications ${ }^{1}$ or in novel several devices as evanescent sensors, filters, optical attenuators, etc. In order to demonstrate the potentiality of these types of structures, a preliminary humidity sensor has been also fabricated.

\section{Acknowledgments}

This work was supported by FEDER and Spanish ClCyT TIC2003-00909 and Gobierno de Navarra Research Grants.

\section{References}

1. K. Oh, S. Choi, Y. Jung, and J. W. Lee, "Novel hollow optical fibers and their applications in photonic devices for optical communications," J. Lightwave Technol. 23(2), 524-532 (2005).

2. Y. Jung, S. Kim, D. S. Lee, and K. Oh, "Compact three segmented multimode fiber modal interferometer for high sensitivity refractiveindex measurement," Proc. SPIE 5855, 150-153 (2005).

3. J. Sirkis, T. A. Berkoff, R. T. Jones, H. Singh, A. D. Kersey, E. J. Friebele, and M. A. Putnam, "In-line fiber etalon (ILFE) fiber-optic strain sensors," J. Lightwave Technol. 13, 1256-1263 (1995).

4. S. Choi, T. J. Eom, Y. Jung, B. H. Lee, J. W. Lee, and K. Oh "Broad-band tunable all-fiber bandpass filter based on hollow optical fiber and long-period grating pair," Photon. Tech. Lett. 17(1), 115117 (2005).

5. Y. Jeong, B. Yang, B. Lee, H. S. Seo, S. Choi, and K. Oh, "Electrically controllable log-period liquid crystal fiber gratings," IEEE Photon. Tech. 12(5), 519-521 (2000)

6. S. Choi, K. Oh, W. Shin, C. S. Park, U. C. Paek, K. J. Park, Y. C. Chung, G. Y. Kim, and Y. G. Lee, "Novel mode converter based on hollow optical fiber for gigabit LAN communication," IEEE Photonics Technol. Lett. 14(2), 248-250 (2002).

7. I. Del Villar, I. R. Matias, F. J. Arregui, and R. O. Claus, "ESA based in-fiber nanocavity for hydrogen peroxide detection," IEEE Trans. Nanotechnol. 4, 187-193 (2005).

8. J. Moreno, F. J. Arregui, and I. R. Matias, "Fiber optic ammonia sensing employing novel thermoplastic polyurethane membranes," Sens. Actuators B 105(2), 419-424 (2005).

9. I. R. Matias, C. Fernández-Valdivielso, F. J. Arregui, C. Bariáin, and M. López-Amo, "Transmitted optical power through a tapered single mode fiber under dynamic bending effects," Fiber Integr. Opt. 22(3), 173-187 (2003).

10. F. J. Arregui, K. L. Cooper, Y. J. Liu, I. R. Matias, and R. O. Claus, "Optical fiber humidity sensor with a fast response time using the ionic self-assembly method," IEICE Trans. Electron. E83-C(3), 360365 (2000). 